\title{
Discovery potential of hidden charm baryon resonances via photoproduction
}

\author{
Yin Huang ${ }^{1,2,3}$, and Jun He $\mathrm{H}^{1,2,4} \dagger$, Hong-Fei Zhang ${ }^{3}$ and Xu-Rong \\ Chen $^{1}$ \\ ${ }^{1}$ Institute of modern physics, Chinese Academy of Sciences, Lanzhou 730000, China \\ ${ }^{2}$ Research Center for Hadron and CSR Physics, Institute of Modern Physics of CAS \\ and Lanzhou University, Lanzhou 730000, China. \\ ${ }^{3}$ School of Nuclear Science and Technology, Lanzhou University, Lanzhou 730000, \\ China \\ ${ }^{4}$ State Key Laboratory of Theoretical Physics, Institute of Theoretical Physics, \\ Chinese Academy of Sciences
}

\begin{abstract}
In this work, we study the possibility to find $N_{c \bar{c}}^{*}$ and $\Lambda_{c \bar{c}}^{*}$ resonances with hidden charm with mass above $4 \mathrm{GeV}$ in the photon-induced production. The cross sections for the photoproductions of hidden charmed baryons are predicted in the effective Lagrangian approach with the vector meson dominance mechanism. The $N^{*}(4412)$ can be produced directly by photon excitation with total cross section about $1 \mathrm{nb}$. The $N^{*}(4412)$ provides a obvious peak near threshold for $J / \psi$ photoproduction, which is promising to be checked by the future high precision experiment at JLab $12 \mathrm{GeV}$. The results will be helpful to the experimental search for the hidden-charmed baryon resonances in the coming experiment at JLab $12 \mathrm{GeV}$, such as SoLID, and the proposed electron-ion colliders at FAIR and HIAF.
\end{abstract}

Keywords: hidden charm baryon resonances, photoproduction, SoLID

$\ddagger$ Corresponding author: junhe@impcas.ac.cn 


\section{INTRODUCTION}

Searching for the exotic hadrons, which are the states beyond the scheme of the conventional quark model with the figures $q \bar{q}$ and $q q q$, is one of the main aims of the hadron physics. The most promising candidates of the exotic states are the puzzled charmonium-like states $X Y Z$ observed in recent years, which are difficult to be put into the frame of the conventional $c \bar{c}$ state [1]. There exist various explanations about such states, such as multiquark state, molecular states. In the baryon side, historically the strange baryon $\Lambda(1405)$ was suggested to be a molecular as $N \bar{K}$ [2]. Recently, the molecular state composed of charmed meson and nucleon is discussed in the one-bosonexchange model [3, 4, 5, 6]. The hidden charm baryon resonances are also suggested in the literature [7, 8, 9, 10, 11, 12]. In this work we will focus on the hidden charmed baryon resonances.

In the conventional quark model, a baryon is made of three constituent quarks. The lowest nucleon resonance with spin-parity $J^{P}=1 / 2^{-}$should be the first $L=1$ orbital excitation state while the experiment suggests the $N^{*}$ (1535) has higher mass than the lowest $J^{P}=1 / 2^{+}$radial excitation state $N^{*}(1440)$. Moreover, the experimental measurements about strange magnetic moment and strange form factor of the proton also indict that the strange quark may play an important role in the nucleon [13, 14]. Zou et al. proposed to include the pentaquark components as qqqs $\bar{s}$ in excited baryons to solve such puzzles [15]. It is natural to expect that there exists baryons with hidden charm if the excited energy becomes larger so that the $c \bar{c}$ appears instead of $s \bar{s}$. Wu et al. predicted the narrow hidden charmmed bayon resonances $N_{\bar{c} c}^{*}$ and $\Lambda_{\bar{c} c}^{*}$ with masses around 4.2-4.6 GeV and widths smaller than $100 \mathrm{MeV}$, which are generated from $P B \rightarrow P B$ and $V B \rightarrow V B$ coupled-channel systems with $P$ and $V$ the pseudoscalar and vector mesons of the 16-plet of SU(4), respectively [7]. Since only S-wave is considered, the angular momentum and parity $J^{P}$ is set as $1 / 2^{-}$in the original papers by Wu et al. [7, 9]. Here we recall the predicted charmed baryon resonances and their decay pattern in Table 1 .

It is interesting to search for such resonances in experiments with the help of the partial decay width predicted. In the original article by $\mathrm{Wu}$ et al., the experimental search in the proton-antiproton collision at PANDA has been suggested [7]. On the other side, it should be an ideal channel to produce the hidden charm baryon resonances through the photon excitation by dragging out a pair of $c \bar{c}$ from the nucleon. The

photoproduction of the charmonium-like states, such as $Z(4430)$ and $Y(3940)$ have been proposed and considerable cross sections are predicted [16, 17]. There exist many facilities in the world to study the normal nucleon resonances, such as CLAS, MAMI, GRAAL and LEPS. However, all these facilities run at too low energy to study hidden charm baryon resonances. The proposed eRHIC, MEIC and LHeC run at too high energy. Fortunately, the JLab $12 \mathrm{GeV}$ with high luminosity just works in the energy region we require. Besides, the proposed electron-ion colliders with lower energy, such as ENC@FAIR in Germany and EIC@HIAF in China, can be used to study the hidden 
Table 1. The charmed baryon resonances and their decay pattern predicted in Ref. [7] with unit of $\mathrm{MeV}$. The first row is for the generated channel. The third and fourth lines are for the masses $M$ and total widths $\Gamma$, respectively. The $\Gamma_{i}$ is the partial decay width of the corresponding charmed baryon resonance.

\begin{tabular}{|c|c|c|c|c|c|c|c|c|c|c|}
\hline & \multicolumn{5}{|c|}{$P B \rightarrow P B$} & \multicolumn{5}{|c|}{$V B \rightarrow V B$} \\
\hline & \multirow{2}{*}{\multicolumn{2}{|c|}{$\frac{N_{c \bar{c}}^{*}}{4261}$}} & & \multicolumn{2}{|c|}{$\Lambda_{c \bar{c}}^{*}$} & \multirow{2}{*}{\multicolumn{2}{|c|}{$\frac{N_{c \bar{c}}^{*}}{4412}$}} & & \multicolumn{2}{|c|}{$\Lambda_{c \bar{c}}^{*}$} \\
\hline$M$ & & & & 4209 & 4394 & & & & 4368 & 4544 \\
\hline$\Gamma$ & & 56.9 & & 32.4 & 43.3 & & 47.3 & & 28.0 & 36.6 \\
\hline \multirow{6}{*}{$\Gamma_{i}$} & $\pi N$ & 3.8 & $K N$ & 15.8 & 0.0 & $\rho N$ & 3.2 & $K^{*} N$ & 13.9 & 0.0 \\
\hline & $\eta N$ & 8.1 & $\pi \Sigma$ & 2.9 & 10.6 & $\omega N$ & 10.4 & $\rho \Sigma$ & 3.1 & 8.8 \\
\hline & $\eta^{\prime} N$ & 3.9 & $\eta \Lambda$ & 3.2 & 7.1 & $K^{*} \Sigma$ & 13.7 & $\omega \Lambda$ & 0.3 & 9.1 \\
\hline & $K \Sigma$ & 17.0 & $\eta^{\prime} \Lambda$ & 1.7 & 3.3 & & & $\phi \Lambda$ & 4.0 & 0.0 \\
\hline & & & $K \Xi$ & 2.4 & 5.8 & & & $K^{*} \Xi$ & 1.8 & 5.0 \\
\hline & $\eta_{c} N$ & 23.4 & $\eta_{c} \Lambda$ & 5.8 & 16.3 & $J / \psi N$ & 19.2 & $J / \psi \Lambda$ & 5.4 & 13.8 \\
\hline
\end{tabular}

charm baryon resonances. In Ref. [32, the generation of the bound state $\mathrm{J} / \psi-{ }^{3} \mathrm{He}$ is investigated in the photoproduction. In this work, we will study the potential to find out such resonances in the photon-nucleon interactions to give the helpful information for the future experimental research.

The paper is organized as follows. After the introduction, we will present the formalism and ingredients used in our calculation. Then in Section III, we will give the numerical results of the calculation, and the possible background is also discussed. The discussion and summary will be given in the last section.

\section{Formalism for the photoproduction}

In this work we will study the photoproduction of the hidden charm baryon $B_{c \bar{c}}^{*}$ including $N_{c \bar{c}}^{*}$ and $\Lambda_{c \bar{c}}^{*}$, which can be divided into two types as shown in Table 1, these decaying decay to a vector meson and a baryon, and these decaying to a peseudoscalar meson and a baryon. For the former the $N_{c \bar{c}}^{*}$ can be produced by vector meson dominance (VMD) mechanism shown in Fig. 1 (a). The latter should be produced by a $t$ channel mechanism as shown in Fig. 1 (b).

In the following, we will present the Lagrangians used in the calculation. In the model used in Ref. [7], only S-wave is considered in the prediction of the mass and decay pattern of hidden charmed baryon resonances. Here we adopt the Lorentz covariant orbit-spin (L-S) scheme for the $B_{c \bar{c}}^{*}$ decays [18], with which only S-wave decay is involved in the Lagrangian. In Ref. [19], Xie et al. found the L-S Lagrangian with VMD mechanism can give more consistent coupling constant of $N^{*}(1535) N \rho$ with L-S coupling than the vector and tensor forms of coupling which involve both S-wave and D-wave decays. This scheme has also been used in Refs. [9, 20] to study the production of $B_{c \bar{c}}^{*}$ with $J^{P}=\frac{1}{2}^{-}$in electroproduction. The explicit forms of the L-S Lagrangians 


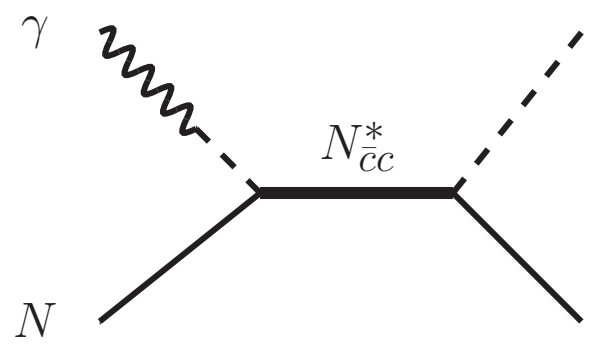

(a)

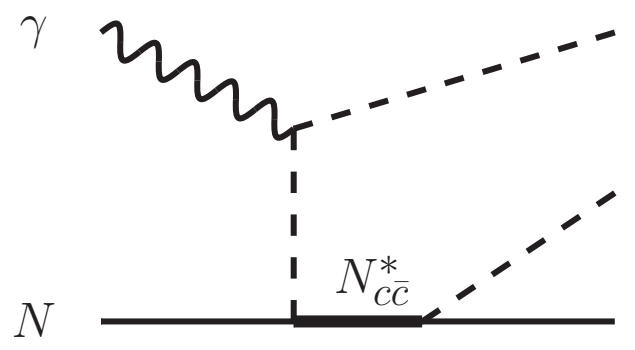

(b)

Figure 1. The Feynman diagrams for the mechanisms of hidden baryon resonance production, (a) direct production through VMD mechanism, (b) production through $t$ channel.

used in this work are

$$
\begin{aligned}
\mathcal{L}_{B_{c \bar{c}}^{*} B P} & =g_{B_{c \bar{c}}^{*} B P} \bar{B}_{c \bar{c}}^{*} P B+\text { h.c. } \\
\mathcal{L}_{B_{c \bar{c}}^{*} B V} & =i g_{B_{c \bar{c}}^{*} B V} \bar{B}_{c \bar{c}}^{*} \gamma_{5} \gamma^{\mu} \tilde{g}^{\mu \nu}(p) V^{\nu} B+\text { h.c. }
\end{aligned}
$$

where $\tilde{g}^{\mu \nu}(p)=\left(g_{\mu \nu}-\frac{p_{\mu} p^{\nu}}{p^{2}}\right)$ with $p$ the momentum of the charmed baryon $B_{c \bar{c}}^{*}$. The explicit values of the couplings for hidden charmed baron $B_{c \bar{c}}^{*}=\left(N_{c \bar{c}}^{*}, \Lambda_{c \bar{c}}^{*}\right)$, baryon $B=$ $(N, \Sigma)$, pseudoscalar meson $P=\left(\pi, \eta, \eta^{\prime}, K\right)$ and vector meson $V=\left(\rho, \omega, J / \psi, K^{*}\right)$ can be found in Table 2. The coupling constants can be determined by the partial decay widths obtained in Ref. [7] as listed in Table 1) The obtained coupling constants which will be used in the following calculation are presented in Table 2.

Table 2. The coupling constant for the decay of $N_{c \bar{c}}^{*}$ or $\Lambda_{c \bar{c}}^{*}$.

\begin{tabular}{cc|cc|cc|cc}
\hline \multicolumn{2}{c|}{$N^{*}(4261)$} & \multicolumn{2}{|c|}{$N^{*}(4412)$} & \multicolumn{2}{c|}{$\Lambda^{*}(4209)$} & \multicolumn{2}{c}{$\Lambda^{*}(4368)$} \\
\hline$\pi N$ & 0.103 & $\rho N$ & 0.030 & $K N$ & 0.369 & $K^{*} N$ & 0.125 \\
$\eta N$ & 0.264 & $\omega N$ & 0.096 & -- & -- & -- & -- \\
$\eta^{\prime} N$ & 0.189 & $J / \psi N$ & 0.416 & -- & -- & -- & -- \\
$K \Sigma$ & 0.224 & $K^{*} \Sigma$ & 0.061 & -- & -- & -- & -- \\
\hline
\end{tabular}

To obtain the radiative decay vertex for $N_{c \bar{c}}^{*}$, the VMD mechanism is introduced. The $V \gamma$ coupling is described as,

$$
L_{V \gamma}=\sum_{V} \frac{e M_{V}^{2}}{f_{V}} V_{\mu} A^{\mu},
$$

where $M_{V}$ is the mass of the vector meson. $V$ and $A$ are the vector meson and electromagnetic fields. As suggested in Ref. [19], the gauge invariance of the diagram in Fig. 1 (a) can be guaranteed by the propagator of the vector meson in VMD mechanism.

There are several ways to determine the coupling constants $e / f_{V}$. In this work, we derive the coupling constants with the experimental partial decay width $\Gamma_{V \rightarrow e^{+} e^{-}}$as listed in Table 3. In the hidden-gauge approach [22, 24], the coupling constants can be related to tensor formalism [23]. In their work, the $S U(4)$ symmetry-breaking effects are 
included by using $g=M_{J / \psi} /\left(2 f_{\eta_{c}}\right)$ for $\gamma-J / \psi$ coupling. The values are also presented in Table 3 ,

Table 3. The coupling constant $e / f_{V}$ determined in $V \rightarrow e^{+} e^{-}$, The data for branching rations are from PDG 21]. The values in the third column are from the hidden-gauge approach [24.

\begin{tabular}{ccccc}
\hline $\begin{array}{c}\text { Coupling constant } \\
e / f_{V}\end{array}$ & $\begin{array}{c}\text { Values } \\
\left(\times 10^{-2}\right)\end{array}$ & $\begin{array}{c}\text { Ref. 24] } \\
\left(\times 10^{-2}\right)\end{array}$ & $\begin{array}{c}\text { Total width } \\
(\mathrm{MeV})\end{array}$ & $\begin{array}{c}B R\left(V \rightarrow e^{+} e^{-}\right) \\
\times 10^{-5}\end{array}$ \\
\hline$e / f_{\rho}$ & 4.986 & 5.173 & $149.1 \pm 0.8$ & $4.72 \pm 0.05$ \\
$e / f_{\omega}$ & 1.459 & 1.698 & $8.49 \pm 0.08$ & $7.28 \pm 0.14$ \\
$e / f_{J / \psi}$ & 2.209 & 3.873 & $(92.9 \pm 2.8) \times 10^{-3}$ & $(5.94 \pm 0.06) \times 10^{3}$ \\
\hline
\end{tabular}

For the diagram (b) in Fig 1, a typical effective Lagrangians for the $\gamma P P$ and $V \gamma P$ couplings are introduced in the following form,

$$
\begin{aligned}
& L_{\gamma \pi \pi}=-i e\left[\pi \partial_{\mu} \pi^{+}-\pi^{+} \partial_{\mu} \pi\right] A_{\mu}, \\
& L_{V \gamma P}=g_{V \gamma P} \epsilon_{\mu \nu \alpha \beta} \partial^{\mu} V^{\nu} \partial^{\alpha} A^{\beta} P
\end{aligned}
$$

where $A, V$ and $P$ are electromagnetic, vector meson and pseudoscalar fields, $M_{V}$ is the vector meson mass, $\epsilon_{\mu \nu \alpha \beta}$ is the anti-symmetric Levi-Civita tensor. The coupling constants $g_{K^{*+} K^{+\gamma}}=0.254 \mathrm{GeV}^{-1}$ and $g_{\rho^{+} \pi^{+} \gamma}=0.222 \mathrm{GeV}^{-1}$ are extracted from the experimental decay width [21].

To include the off-shell effect, two types of form factors for meson and baryon in the $B B M$ vertices are introduced,

$$
F_{M}\left(q^{2}\right)=\frac{\Lambda_{M}^{2}-m_{M}^{2}}{\Lambda_{M}^{2}-q^{2}}, \quad F_{B}\left(q^{2}\right)=\frac{\Lambda_{B}^{4}}{\Lambda^{4}+\left(q^{2}-m_{B}^{2}\right)^{2}},
$$

where the $m_{(M, B)}$ is the mass of the off-shell meson or baryon. In this work the cut off $\Lambda_{M}$ is chosen as $\Lambda_{M}=m_{M}+\alpha \Lambda_{\mathrm{QCD}}$ with $\Lambda_{\mathrm{QCD}}=0.22 \mathrm{GeV}$. The parameter $\alpha$ is close to unitary usually. In this work, we choose $\alpha=1.3$. The cutoff for the off-shell baryon is chosen as $\Lambda_{B}=1 \mathrm{GeV}$. In this work we will also discuss the effect of the uncertainties of the cut off by variation $\alpha$ from to 2 .

\section{Numerical results}

In this section we will study the production of the hidden charmed baryon with the Lagrangians given in the previous section with the possible background from the experiment or theoretical prediction.

\subsection{The photoproduction of $N^{*}(4412)$}

Since $N^{*}(4412)$ decays to $N / \Sigma$ and a vector meson mainly, the photon can excite the target nucleon to the hidden charmed nucleon resonance $N^{*}(4412)$ through VMD mechanism as shown in Fig 1 (a). The intermediated vector mesons in the VMD 
mechanism are the neutral vector meson $\rho^{0}, \omega$ and $J / \psi$. Due to the off-shellness of the intermediate vector mesons, the form factor should be added to the corresponding vertices [25], which will lead to the suppression of $J / \psi$ exchange contribution.

As shown in Table 1, $N^{*}(4412)$ only couples to $V B$, which is due to the exclusion of the $V B \rightarrow P B$ transition in Ref. [7] The $V B \rightarrow P B$ transition would involve pseudoscalar exchange. However, the studies in the light sector for baryon resonances [26, 27] and in the heavy sector for hidden charm mesons have been done concluding that this effect would be small [28, 29]. Hence we do not consider this correction in this work. Other possibility is a contact term such as the KrollRuddermann term studied in Refs. [26, 27]. It should a background contribution which will be included in two-gluon and three gluon exchange contributions.

The near threshold behavior of $\gamma p \rightarrow J / \psi p$ process has been studied in the literature [30, 31, 32]. Here we adopt the two gluon $(2 g)$ and three-gluon $(3 g)$ exchanges in Ref. [30] as background. In Ref. [32], the $2 g+3 g$ model can describe the cross section up to an energy about $20 \mathrm{GeV}$ generally. The amplitude adopted is written as [32],

$$
\mathcal{A}=\frac{1}{2 \pi^{2}} \frac{1}{2 E_{J / \psi}(|\boldsymbol{k}|)} \sqrt{\frac{m_{p}}{E_{p}(|\boldsymbol{k}|)}} \sqrt{\frac{m_{p}}{E_{p}(|\boldsymbol{q}|)}} \frac{1}{\sqrt{2|\boldsymbol{q}|}} \frac{4 \sqrt{\pi}}{\sqrt{6}} \frac{|\boldsymbol{q}| W}{m_{p}}\left[\boldsymbol{M}_{2 g}+\boldsymbol{M}_{3 g}\right](7)
$$

with

$$
\boldsymbol{M}_{2 g}=\frac{A_{2 g}}{4 \sqrt{\pi}} \frac{1-x}{R M_{J / \psi}} e^{b t / 2}, \quad \boldsymbol{M}_{3 g}=\frac{A_{3 g}}{4 \sqrt{\pi}} \frac{1}{R^{2} M_{J / \psi}^{2}} e^{b t / 2},
$$

where $E_{J / \psi}(|\boldsymbol{k}|), E_{p}(|\boldsymbol{k}|), E_{p}(|\boldsymbol{q}|)$ are the energies for the $J / \psi$, initial proton and final proton with initial and final momentum $k$ and $q . x=\left(2 m_{p} M_{J / \psi}+M_{J / \psi}^{2}\right) /\left(W^{2}-m_{p}^{2}\right)$, where $W, M_{J / \psi}$ and $m_{p}$ are the energy in center of mass frame, the mass of $J / \psi$ and mass of proton. As in Refs. [30, 32], $R=1 \mathrm{fm}$ and $b=1.14 \mathrm{GeV}^{-2}$. The parameters $A_{2 g}$ and $A_{3 g}$ are determined by fitting the data. With the background and resonance contributions, the cross section can be obtained as shown in Fig. 2.

The old experimental data with large uncertainties, which were obtained in the seventies of the last century, show a peak near the energy point $W=4.412 \mathrm{GeV}$. Althrough the data point about $4.4 \mathrm{GeV}$ is well reproduced in the $2 g+3 g$ model in Ref. [32, the cross sections in the $2 g+3 g$ model are larger than the PM model and experimental data generally. If we decrease the $3 g$ contribution, the experimental data can be well reproduced with almost only the $2 g$ exchange mechanism except the data point at $W=4.4 \mathrm{GeV}$. In Ref. [30], Brodsky et al. suggested that inclusion of the contributions of five-quark resonance near threshold will improve the theoretical results. Here we consider the hidden charm baryon resonance $N^{*}(4412)$, which gives a narrow peak at energies about $4.4 \mathrm{GeV}$ as shown in Fig. 2. To fit the experimental data, the $3 g$ contribution should be suppressed by adjusting the free parameter $A_{3 g}$. After including the $N^{*}(4412)$, the $2 g$ contribution is dominant to reproduce the experimental data. Hence we consider the $2 g$ contribution only in this work and determine the parameter $A_{2 g}$ with the experimental data except the data point around $4.4 \mathrm{GeV}$. It is still possible that the $3 g$ contribution is dominant very near threshold (such as lower than $4.2 \mathrm{GeV}$ ), 


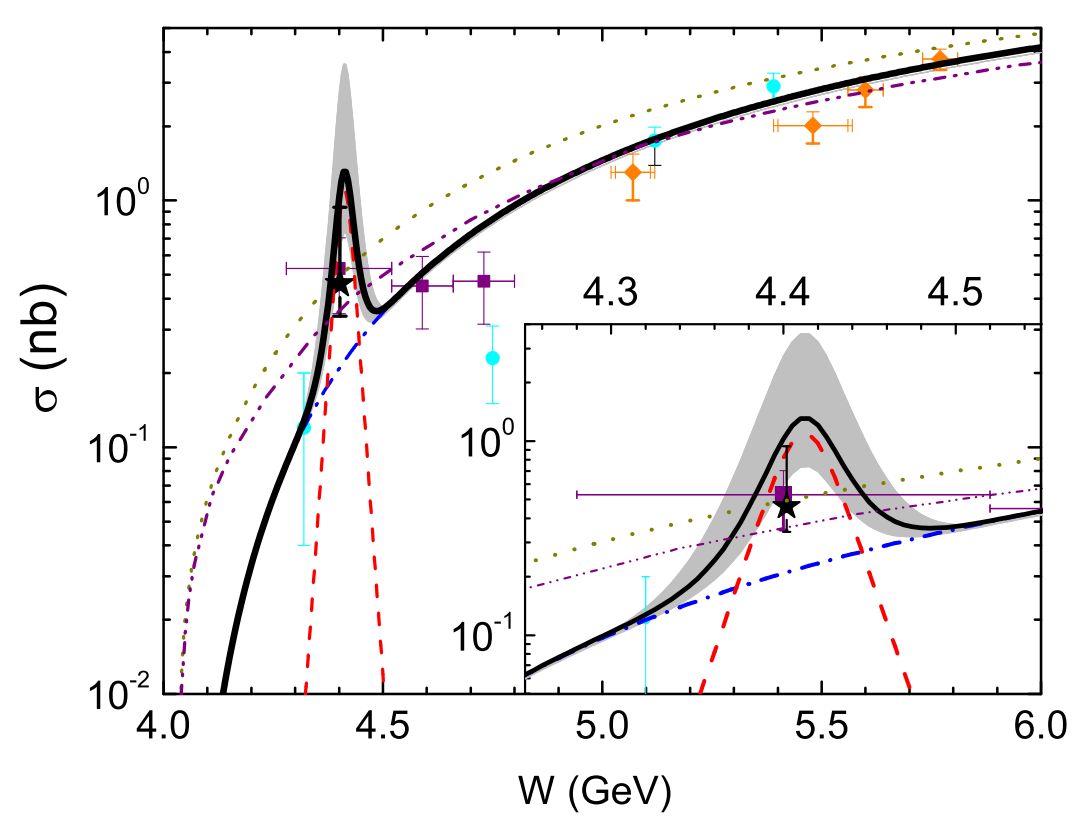

Figure 2. (Color online) The cross sections for $J / \psi$ photoproduction as function of the energy $W$ in center of mass frame. The full (black), dashed (red) dash-dotted (blue) are for total, $N^{*}(4412)$ and back ground contributions. The band is for the total cross section with the variation of $\alpha$ in the cut off $\Lambda$ from 1 to 2 . The star is explained in the text. The $2 g+3 g$ model and PM model results in Ref. 32] are also shown as dotted (dark yellow) and dash-dot-dotted (purple) lines.The subfigure focus on the energies around $W=4.412 \mathrm{GeV}$, and the position of the star is moved to the right slightly to avoid overlapping with the experimental data from Cornell. The experimental data are from SLAC 75 [33] (square), SLAC 76 [34] (diamond) and Cornell [35] (circle).

which is out of the interesting energy scope in the current work. In Ref. [32], the PM model is fitted to all data points around $4.4 \mathrm{GeV}$ without explicit mechanism at energies around $4.4 \mathrm{GeV}$. Hence, though there are some differences at low energies, our result is not in conflict with the PM model.

The contribution from the charmed baryon $N^{*}(4412)$ can be calculated from the predicted decay width in Ref. [7] and Lagrangians presented in the previous section. Due to the narrow width of $N^{*}(4412)$, we give the total cross section in the corresponding bin of the Cornell experiment to make comparison. The result shows that after including the contribution of the $N^{*}(4412)$ to the background contribution, the theoretical result with $\alpha=1.3$ is close to the experimental one. However the large uncertainty of the existing data make it difficult to give a confirmative conclusion. The results are not sensitive to the cut off $\Lambda_{B}$. To present the effect of the variation of the parameters $\Lambda_{M}=m_{M}+\alpha \Lambda_{Q C D}$, the results with $1<\alpha<2$ are also shown in the Fig. 2. The theoretical results are consistent with the experimental data considering the uncertainty of the experiment. The confirmation of the existence of $N^{*}(4412)$ need high precision experiment. The total cross section through intermediating $N^{*}(4412)$ is about $1 \mathrm{nb}$ 
at the energy point $W=4.412 \mathrm{GeV}$. An experiment with precision about $0.1 \mathrm{nb}$ will be enough to check the existence of the hidden-charmed baryon $N^{*}(4412)$. The future experiment in JLab $12 \mathrm{GeV}$ with large luminosity is promising to reach such requirement [36].

In the former results the total cross sections are given, now we present the differential cross sections at the energy point $W=4.412 \mathrm{GeV}$ in Fig. 3. It is well known that the differential cross section $d \sigma / d t$ will decrease exponentially as suggested by Eq. (8). The existence of the $N^{*}(4412)$ will change the feature of the differential cross section completely. As shown in Fig. 3, the differential cross section $d \sigma / d t$ is weakly dependent of Mandelstam variable $-t$. Since $-t$ is in proportional to the outgoing angle $\theta$ at certain $W$, the differential cross section $d \sigma / d \cos \theta$ is also weakly dependent of angle $\theta$ in the center of mass frame. Hence, it is an excellent way to search for the charm baryon resonance $N^{*}(4412)$ by measuring the differential cross sections at the energy point $W=4.412 \mathrm{GeV}$ with higher $-t$.

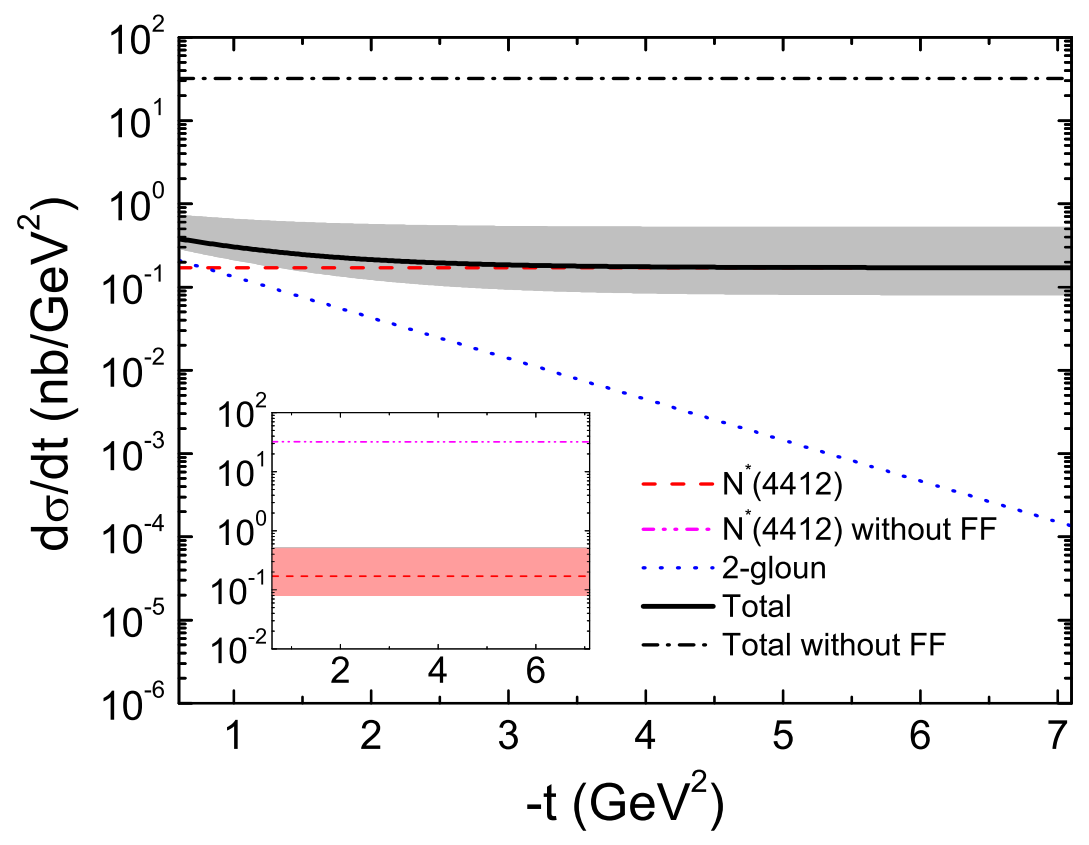

Figure 3. (Color online) The differential cross sections for $J / \psi$ production as function of $-t$ in center of mass frame. The full, dashed and dotted lines are for the total, $N^{*}(4412)$ and $2 g$ contributions, respectively. The grey band is for total contribution with the variation of $\alpha$ from 1 to 2 . The subfigure is for the $N^{*}(4412)$ contribution with $1<\alpha<2$. The dash-dotted and dash-dot-dotted lines are for the total and $N^{*}(4412)$ contributions without from factor $(\mathrm{FF})$.

We also present the results without the form factors to show the uncertainties tied to the use of the form factors. The results show that the form factor can lead to the suppression of the cross section by two orders of magnitude. If the form factors 
are removed, there are large deviations from the experimental data even if the large uncertainties of the data are considered. Hence, the inclusion of the form factors is necessary in our effective Lagrangian approach.

Besides the $J / \psi p$ channel, we will consider the $K \Sigma$ channel. In the $K \Sigma$ channel there are no experimental data in the energy region near $W=4.412 \mathrm{GeV}$ to our knowledge. There exist many theoretical studies [37, 38, 39. To estimate the background, we extend the theoretical results for the $\gamma p \rightarrow K^{* 0} \Sigma^{+}$and $\gamma p \rightarrow K^{*+} \Sigma^{0}$ by the model of Nam et al. [40] to the higher energy region as the background for the hidden charm $N^{*}$ (4412). At the low energies considered in Ref. [40, the dominant contributions are from $K-, \kappa$-exchanges and $\Delta$. At energies higher than $4 \mathrm{GeV}$, the contributions from s-channel, that is, the contributions from the normal nucleon resonances, should be negligible because their masses are below $3 \mathrm{GeV}$. The t-channel contributions from $K^{*}, K$ and $\kappa$ exchanges are dominant and the contribution from $\Delta$ is negligible. For the neutral $K^{*}$ photoproduction, the contact and $K^{*}$ exchange contributions are absent. For the charged $K^{*}$ photoproduction, the contact contribution decreases slowly and can provide considerable contribution as shown in Fig. 4 .

The background contributions at the energy point $W=4.412 \mathrm{GeV}$ are about $20 \mathrm{nb}$ and $6 \mathrm{nb}$ for the $\gamma p \rightarrow K^{* 0} \Sigma^{+}$and $\gamma p \rightarrow K^{*+} \Sigma^{0}$ processes, respectively. As shown in

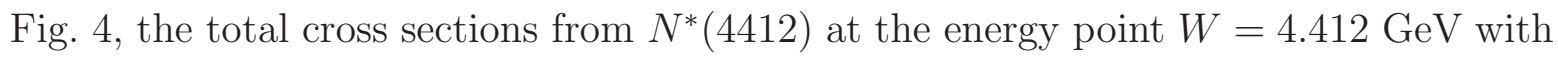
$\alpha=1.3$ are $0.4 \mathrm{nb}$ and $0.2 \mathrm{nb}$ for $K^{* 0} \Sigma^{+}$channel and $K^{*+} \Sigma^{0}$ channel, respectively. With the variation of the cut off, the total cross section from the $N^{*}(4412)$ is in the order 0.1-1 nb. Compared with the $J / \psi p$ channel, the total cross section from $N^{*}(4412)$ is smaller while the background is larger. The precision required in this channel is of the order of $0.01 \mathrm{nb}$. Hence the requirement to observe $N^{*}(4412)$ in this channel is higher than that in $J / \psi p$ channel.

The total cross sections of $N^{*}(4412)$ photoproductions in the $\rho p$ and $\omega$ channels can be calculated analogously. In the $\rho p$ channel the total cross section is about one order of magnitude smaller than the $J / \psi p$ and $K \Sigma$ channels, which results in that the accuracy required in the search for $N^{*}(4412)$ in the $\rho p$ channel is very high. The total cross section for the $\omega p$ channel is of the same order as the channels $J / \psi p$ and for $K \Sigma$. There exists some old experimental data in the energy region considered in this work. The SLAC experiment done in the seventies of last century measured the total cross section at the photon energy $E_{\gamma}=9.3 \mathrm{GeV}$, that is, the energy in center of mass frame $W=4.3 \mathrm{GeV}$, and values about $20 \mu \mathrm{b}$ and $5 \mu \mathrm{b}$ are obtained [43. Such large background make the search for $N^{*}(4412)$ very difficult considering the small produced cross section predicted in the current work. Hence we do not suggest to search $N^{*}(4412)$ in $\gamma p \rightarrow \rho p$ and $\gamma p \rightarrow \omega p$ channels.

\subsection{The photoproduction of $N^{*}(4261)$}

Now we turn to another predicted hidden charm nucleon resonance, $N^{*}(4261)$, which decays to a pseudoscalar meson and a baryon mainly. Due to that a vector meson is 


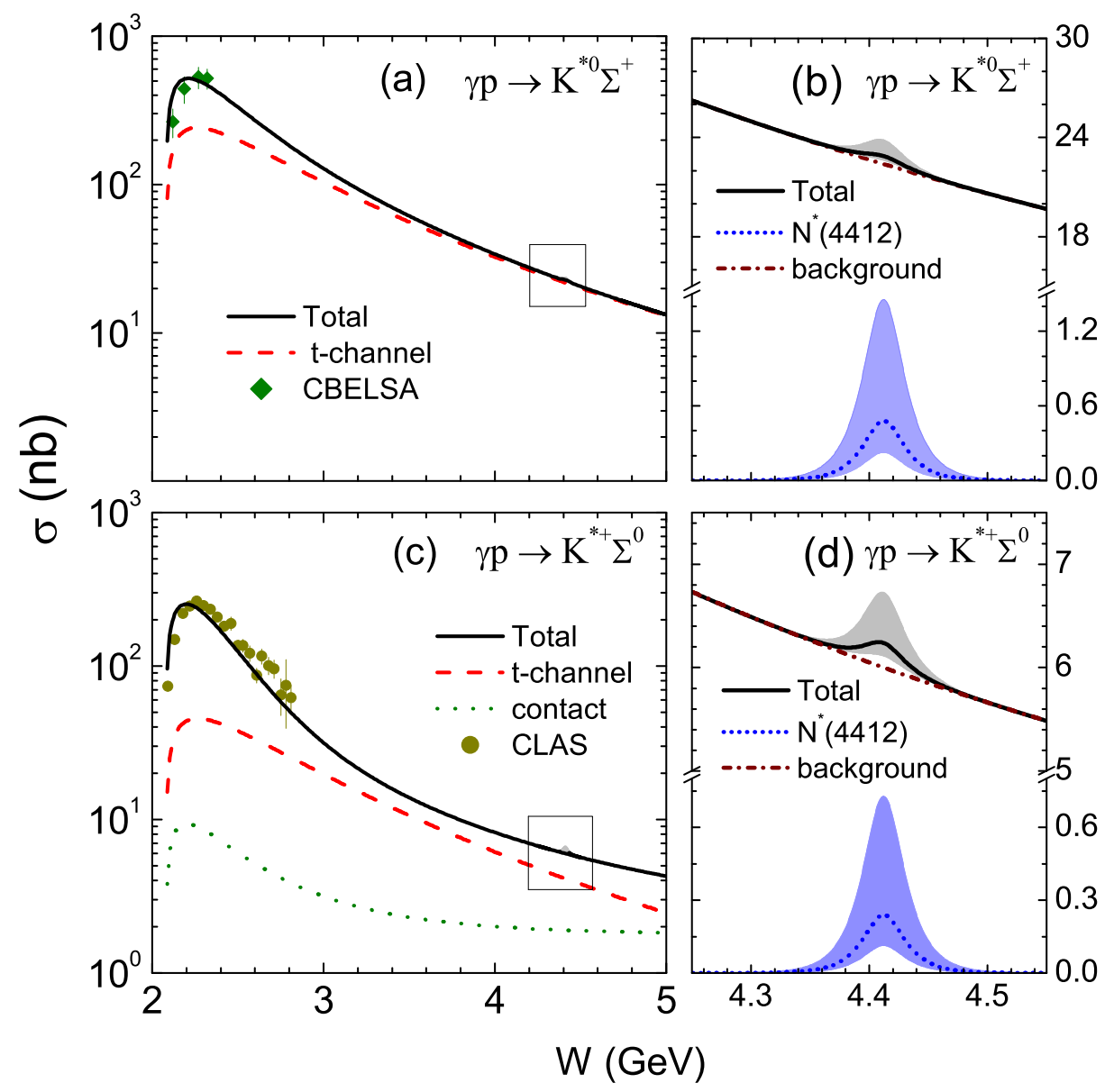

Figure 4. (Color online) The total cross sections for $\gamma p \rightarrow K^{*+} \Sigma^{0}$ and $\gamma p \rightarrow K^{* 0} \Sigma^{+}$ as function of the energy $W$ in the center of mass frame. The figures (a) and (c) are for the cross sections at the energies from threshold to $5 \mathrm{GeV}$ of proceeses $\gamma p \rightarrow K^{*+} \Sigma^{0}$ and $\gamma p \rightarrow K^{* 0} \Sigma^{+}$, respectively. The figures (b) and (d) focus on the energies near the $N^{*}(4412)$ pole. And the contribution of $N^{*}(4412)$ is presented in figures (c) and (d) as a dotted line. The grey and light blue bands are for total and $N^{*}(4412)$ contributions. The experimental data are from Refs. [41, 42.

essential in the VMD mechanism, $N^{*}(4261)$ can not be produced by photon excitation directly. It should be produced through the mechanism shown in Fig 1(b). Considering that the $\pi$ meson is easy to be detected in the experiment we consider the final state with $\pi$ and $N^{*}(4261) \rightarrow B P$ only. Since the vertex $\gamma \pi^{0} \pi^{0}$ does not exist, the only possible channel is $\gamma p \rightarrow \pi^{+} N_{c \bar{c}}^{*} \rightarrow \pi^{+} B P$ as shown in Fig. 5 (a). However the amplitude with only $t$ channel contribution does not satisfy the gauge invariance. The $s$ channel contribution as shown in Fig. (5) (b) is included to restore the gauge invariance. Compared with the $N^{*}(4412)$ which can be produced by the photon excitation directly through VMD mechanism, the possibility for the production of $N^{*}(4261)$ with three body final state should be smaller due to the suppression of the phase space.

First, we consider the total cross section for the $N^{*}(4261)$ production, $\gamma p \rightarrow$ 


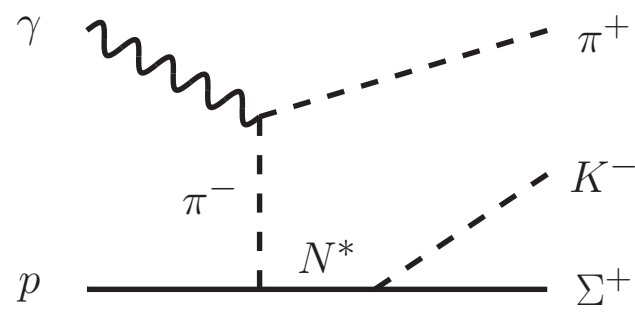

(a)

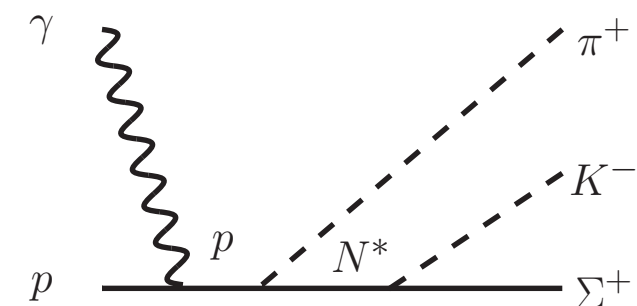

(b)

Figure 5. The diagram for $\gamma p \rightarrow \pi^{+} K^{-} \Sigma^{+}$. Figures (a) and (b) are for t-channel and s-channel, respectively.

$\pi^{+} N^{*}(4261)$. As shown in Fig. 6, the total cross sections are in the order 0.01-0.1 nb which are about one oder of magnitude lower than the total cross sections of $N^{*}(4412)$. The total cross sections for the channels $\pi^{+} K \Sigma$ and $\pi^{+} \eta_{(c)}^{(\prime)} N$ can be estimated from the total cross section for the $N^{*}(4261)$ production with the help of the branch ratio of the corresponding decay channel. Here we take the channel $\gamma p \rightarrow \pi^{+} K^{-} \Sigma^{+}$as an example to make an explicit calculation.In Fig. 6, total cross section from $N^{*}(4261)$ is of the order of $0.001 \mathrm{nb}$. In the literature [44, 45, 46, there existed some old data for $\gamma p \rightarrow \pi K \Sigma$, which are at the order of $\mu b$. It is difficult to search for the $N^{*}(4261)$ in the experiment with so large background.

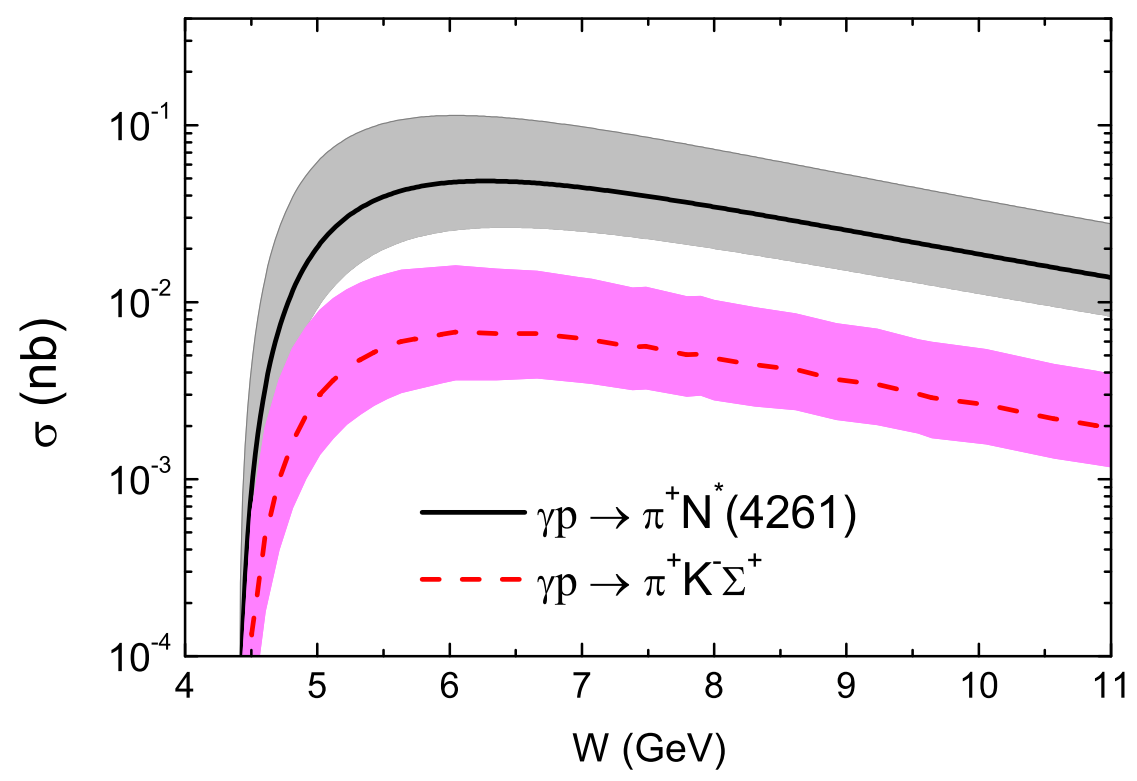

Figure 6. (Color online) The total cross sections for photoproduction of $N^{*}(4261)$ as a function of the energy $W$ in center of mass frame. The full and dashed lines are for the total cross section of $\gamma p \rightarrow \pi^{+} N^{*}(4261)$ and $\gamma p \rightarrow \pi^{+} K^{-} \Sigma^{+}$channel. The bands are for the uncertainties with the variation of $\alpha$ from 1 to 2 . 


\subsection{The photoproduction of $\Lambda_{\bar{c} c}^{*}$}

The hidden charm strange baryons $\Lambda_{\bar{c} c}^{*}$ are also predicted in Refs. [7, 8]. $\Lambda^{*}(4544)$ decays to a vector meson and strange baryon or a $K^{*}$ meson and a nucleon. The VMD mechanism connect the vector with photon, so a strange baryon is essential in the initial state to produce $\Lambda^{*}(4544)$. Considered the $K^{*}$ meson can not be connected to a photon, $\Lambda^{*}(4544)$ can not be produced in $\gamma p$ collision through the mechanism in Fig 1(a). For the mechanism in Fig 1(b) the exchanged meson should be a strange meson for $\gamma p$ collision. The zero decay width of $\Lambda^{*}(4544)$ in $K^{*} N$ channel exclude such production channel. Similarly, $\Lambda^{*}(4394)$ can not be produced through both mechanisms. $\Lambda^{*}(4209)$ and $\Lambda^{*}(4368)$ can be produced by mechanism (b).

The thresholds of $\Lambda^{*}(4209)$ and $\Lambda^{*}(4368)$ productions are higher than the energy region of JLab $12 \mathrm{GeV}$, but may be reached by the proposed EICs at HIAF and FAIR. Here we present our results for the future possible experiments. The cross sections of the productions of $\Lambda_{c \bar{c}}^{*} \mathrm{~s}$ are plotted in Fig. 7. The total cross sections of $\Lambda^{*}(4209)$ and $\Lambda^{*}$ (4368) productions are of the order of $10^{-3} \mathrm{nb}$, which are smaller than these of hidden charm nucleon resonances $N^{*}(4261)$ production because the exchanged mesons $K$ and $K^{*}$ here are heavier than the exchanged meson $\pi$ in $N^{*}(4261)$ production .

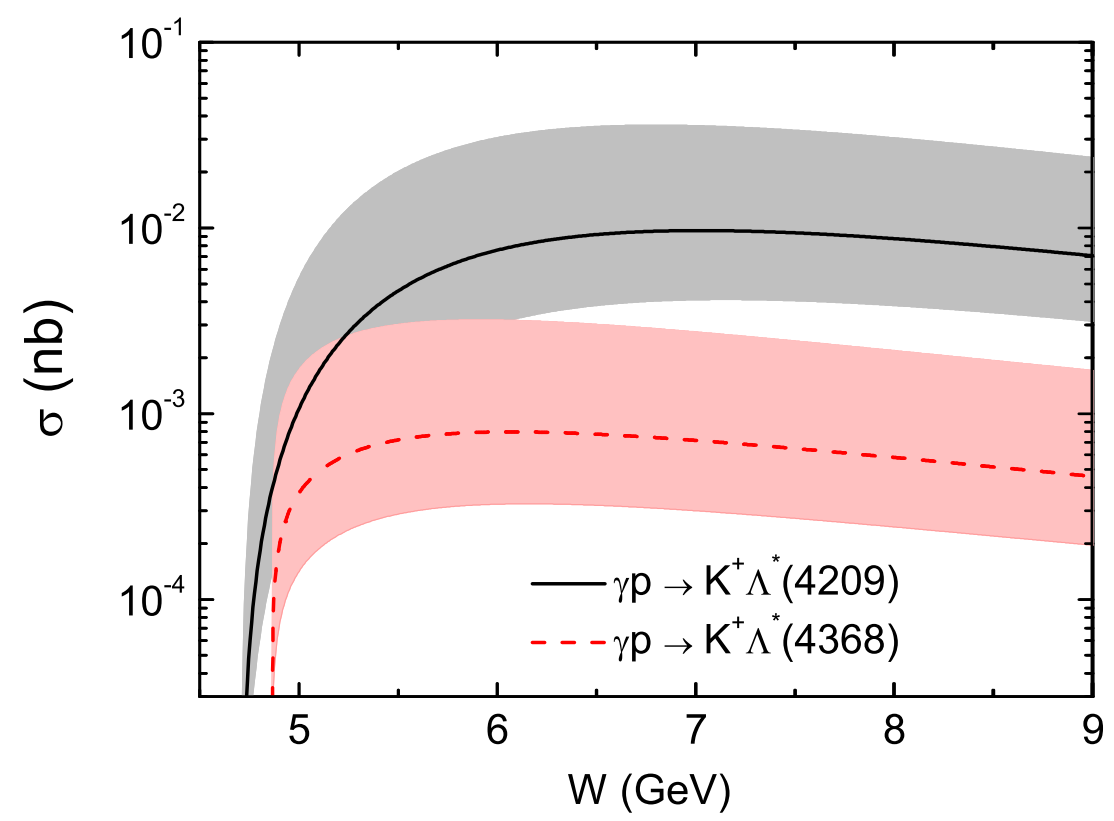

Figure 7. (Color online) The total cross sections for the productions of $\Lambda^{*}(4209)$ and $\Lambda^{*}(4368)$. The bands sre for the uncertainties with the variation of $\alpha$ from 1 to 2 . 


\section{Summary}

The baryon resonances with hidden charm $B_{c \bar{c}}^{*}$ with mass above $4 \mathrm{GeV}$ have been predicted in several approaches. The JLab $12 \mathrm{GeV}$ and the proposed EICs will provide excellent experimental condition to search for the hidden charm baryon resonances. In this work, the possibility of observation of $D_{c \bar{c}}^{*}$ resonances via photoproduction are studied in the effective Lagrangian approach with VMD mechanism.

A peak around $4.412 \mathrm{GeV}$ can be found near the threshold for $J / \psi$ photoproduction . The theoretical values of the total cross sections of near threshold $J / \psi$ photoproduction through the hidden charmed nucleon resonance $N^{*}(4412)$ is about $1 \mathrm{nb}$. If the precision of the experiment reach the order of $0.1 \mathrm{nb}$, it is promising to observe $N^{*}(4412)$. Such requirement can be reached by JLab after $12 \mathrm{GeV}$ upgrade. Besides, a measurement of the differential cross section with large $t$ around $W=4.4 \mathrm{GeV}$ is suggested.

The total cross section for the $N^{*}(4261)$ production is about one order smaller than the total cross section for $N^{*}(4412)$ with large background. The thresholds for the production of hidden charm strange baryon resonances $\Lambda_{c \bar{c}}^{*}$ are beyond the energy

region of JLab $12 \mathrm{GeV}$ and the total cross sections are in the order $10^{-3} \mathrm{nb}$. Hence, it is difficult to search for these states in JLab and the proposed EICs.

Based on the results above, the search for $N^{*}(4412)$ in the $\gamma p \rightarrow J / \psi p$ channel is most promising. Our results will be helpful to the experimental search for the hiddencharmed baryon resonances in the coming experiment at JLab $12 \mathrm{GeV}$, such as SoLID, and the proposed electron-ion colliders at FAIR and HIAF.

\section{Acknowledgments}

This project is partially supported by the Major State Basic Research Development Program in China (No. 2014CB845405), the National Natural Science Foundation of China (Grants No. 11275235, No. 11035006) and the Chinese Academy of Sciences (the Knowledge Innovation Project under Grant No. KJCX2-EW-N01).

\section{References}

[1] S. Godfrey and S. L. Olsen, Ann. Rev. Nucl. Part. Sci. 58, 51 (2008)

[2] R. H. Dalitz and S. F. Tuan, Annals Phys. 10, 307 (1960).

[3] S. Yasui and K. Sudoh, Phys. Rev. D 80, 034008 (2009)

[4] J. He, X. Liu, Phys. Rev. D 82, 114029 (2010)

[5] J. He, D. -Y. Chen and X. Liu, Eur. Phys. J. C 72, 2121 (2012)

[6] Y. Yamaguchi, S. Ohkoda, S. Yasui and A. Hosaka, Phys. Rev. D 84, 014032 (2011)

[7] J. -J. Wu, R. Molina, E. Oset and B. S. Zou, Phys. Rev. Lett. 105, 232001 (2010)

[8] W. L. Wang, F. Huang, Z. Y. Zhang and B. S. Zou, Phys. Rev. C 84, 015203 (2011)

[9] J. -J. Wu, T. -S. H. Lee and B. S. Zou, Phys. Rev. C 85, 044002 (2012)

[10] Z. -C. Yang, Z. -F. Sun, J. He, X. Liu and S. -L. Zhu, Chin. Phys. C 36, 6 (2012)

[11] S. G. Yuan, K. W. Wei, J. He, H. S. Xu and B. S. Zou, Eur. Phys. J. A 48, 61 (2012)

[12] C. Gobbi, D. O. Riska and N. N. Scoccola, Phys. Lett. B 296, 166 (1992).

[13] D. T. Spayde et al. [SAMPLE Collaboration], Phys. Lett. B 583, 79 (2004) 
[14] F. E. Maas et al., Phys. Rev. Lett. 94, 152001 (2005)

[15] B. S. Zou and D. O. Riska, Phys. Rev. Lett. 95, 072001 (2005)

[16] X. -H. Liu, Q. Zhao and F. E. Close, Phys. Rev. D 77, 094005 (2008)

[17] J. He and X. Liu, Phys. Rev. D 80, 114007 (2009)

[18] B. S. Zou and F. Hussain, Phys. Rev. C 67, 015204 (2003)

[19] J. -j. Xie, C. Wilkin and B. -s. Zou, Phys. Rev. C 77, 058202 (2008)

[20] J. -J. Wu and B. S. Zou, Phys. Lett. B 709, 70 (2012)

[21] J. Beringer et al. (Particle Data Group), Phys. Rev. D86, 010001 (2012)

[22] H. Nagahiro, L. Roca, A. Hosaka and E. Oset, Phys. Rev. D 79, 014015 (2009)

[23] G. Ecker, J. Gasser, H. Leutwyler, A. Pich and E. de Rafael,

[24] T. Branz, R. Molina and E. Oset, Phys. Rev. D 83, 114015 (2011) arXiv:1010.0587 [hep-ph]].

[25] Q. Zhao, G. Li and C. -H. Chang, Phys. Lett. B 645, 173 (2007)

[26] K. P. Khemchandani, A. Martinez Torres, H. Kaneko, H. Nagahiro and A. Hosaka, Phys. Rev. D 84, $094018(2011)$

[27] E. J. Garzon and E. Oset, Eur. Phys. J. A 48, 5 (2012)

[28] R. Molina and E. Oset, Phys. Rev. D 80, 114013 (2009)

[29] J. Nieves and M. Pavon Valderrama, Phys. Rev. D 86, 056004 (2012)

[30] S. J. Brodsky, E. Chudakov, P. Hoyer and J. M. Laget, Phys. Lett. B 498, 23 (2001)

[31] A. Sibirtsev, S. Krewald and A. W. Thomas, J. Phys. G 30, 1427 (2004).

[32] J. -J. Wu and T. -S. H. Lee, Phys. Rev. C 86, 065203 (2012)

[33] U. Camerini et al., Phys. Rev. Lett. 35, 483 (1975).

[34] R. L. Anderson, Excess Muons and New Results in Photoproduction, SLAC-PUB- 1417.

[35] B. Gittelman, K. M. Hanson, D. Larson, E. Loh, A. Silverman and G. Theodosiou, Phys. Rev. Lett. 35, 1616 (1975).

[36] J. Arrington et al. [ATHENNA Collaboration], Near Threshold Electroproduction of $J / \psi$ at 11 GeV, Experiment proposal to JLab-PAC39.

[37] J. P. Ader, M. Capdeville and P. Salin, Nucl. Phys. B 17, 221 (1970).

[38] M. Guidal, J. M. Laget and M. Vanderhaeghen, Nucl. Phys. A 627, 645 (1997).

[39] A. Sibirtsev, J. Haidenbauer, F. Huang, S. Krewald and U. -G. Meissner, Eur. Phys. J. A 40, 65 (2009)

[40] S. -H. Kim, S. -i. Nam, A. Hosaka and H. -C. Kim, $K^{*} \Sigma$ photoproduction off the proton target with baryon resonances, arXiv:1211.6285 [hep-ph].

[41] W. Tang et al. [CLAS Collaboration], Cross sections for the $\gamma p \rightarrow K^{*+} \Lambda$ and $\gamma p \rightarrow K^{*+} \Sigma^{0}$ reactions measured at CLAS, arXiv:1303.2615 [nucl-ex].

[42] M. Nanova et al. [CBELSA/TAPS Collaboration], Eur. Phys. J. A 35, 333 (2008)

[43] J. Ballam et al., Phys. Rev. D 7, 3150 (1973).

[44] R. Erbe et al. [Aachen-Berlin-Bonn-Hamburg-Heidelberg-Muenchen, Collaboration], Phys. Rev. 188, 2060 (1969).

[45] R. Erbe et al. [Aachen-Berlin-Bonn-Hamburg-Heidelberg-Muenchen Collaboration], Nuovo Cimento 49A, 504 (1967).

[46] Cambridge Bubble Chamber Group, Phys. Rev. 156, 1426 (1967). 